\title{
Counting and Addition cannot express Deterministic Transitive Closure
}

\author{
Matthias Ruhl \\ MIT Laboratory for Computer Science \\ Cambridge, MA 02139, USA \\ ruhl@theory.lcs.mit.edu
}

\begin{abstract}
An important open question in complexity theory is whether the circuit complexity class $T C^{0}$ is (strictly) weaker than LOGSPACE. This paper considers this question from the viewpoint of descriptive complexity theory.

$T C^{0}$ can be characterized as the class of queries expressible by the logic $\operatorname{FOC}(<,+, \times)$, which is first-order logic augmented by counting quantifiers on ordered structures that have addition and multiplication predicates. We show that in first-order logic with counting quantifiers and only an addition predicate it is not possible to express "deterministic transitive closure" on ordered structures. As this is a LOGSPACE-complete problem, this logic therefore fails to capture LOGSPACE. It also directly follows from our proof that in the presence of counting quantifiers, multiplication cannot be expressed in terms of addition and ordering alone.
\end{abstract}

\section{Introduction}

The interest in finite model theory from a complexity theory point of view is motivated by the fact that "descriptive complexity theory" allows to rephrase complexity theoretic questions in a purely logical framework $[15,16,17]$. This allows one to attack these questions from a new perspective and using different tools, such as EhrenfeuchtFraïssé-games.

In this paper, we study the expressive power of firstorder logic augmented with counting quantifiers on ordered structures. Expressions in first-order logic are built from relational expressions $R x_{1} x_{2} \ldots x_{r}$ and equalities $x_{1}=x_{2}$ using the connectives $\wedge, \vee, \neg$, and the quantifiers $\exists$ and $\forall$. The limitations of the expressiveness of first-order logic are well-known (see, e.g., [5]), most notably that it lacks any ability to 'count'. It is not even possible to express the fact that a structure has even size.

Adding 'counting quantifiers' to the logic remedies this fact. Counting quantifiers are expressions of the form $\exists^{\geq} x \varphi(x)$ and express that "there are at least $y$ distinct elements $x$ that satisfy $\varphi(x)$ ". There has been a great interest in recent years in the expressiveness of first-order logic augmented with counting quantifiers, which we will call $F O C$ in this paper.

This is mostly due to the fact that this logic, in the presence of an ordering, and addition and multiplication predicates (we call this logic " $F O C(<,+, \times)$ )"), captures the circuit complexity class $T C^{0}$ [3], the class of uniform polynomial-size, constant depth, threshold circuits. It is an important open question whether $T C^{0}$ is weaker than LOGSPACE; even whether it is strictly weaker than NP is unknown [2].

To see whether a logic captures LOGSPACE it is sufficient to determine whether it can express "deterministic transitive closure". Deterministic transitive closure (DTC) is the problem where given a directed graph whose vertices all have out-degree at most 1 , and two nodes $s$ and $t$, one has to decide whether there is a path from $s$ to $t$. DTC is LOGSPACE-complete under quantifier-free reductions [8].

The main result of the present paper is that first-order with counting quantifiers on ordered structures with an addition predicate (we call this logic " $F O C(<,+)$ )") and a binary relation $E$, i.e. on structures of the form

$$
\mathcal{A}=(\{0,1, \ldots, N\},<,+, E),
$$

cannot express the deterministic transitive closure of $E$, and thus fails to capture LOGSPACE.

We prove this main result by giving an explicit winning strategy for the second player in an Ehrenfeucht-Fraïsségame.

From a descriptive complexity theory point of view, it shows that the multiplication predicate must be essential should $F O C(<,+, \times)$ in fact equal LOGSPACE. Moreover, from our proof we can also directly conclude the stronger result that $F O C(<,+) \neq F O C(<,+, \times)$ meaning that multiplication is essential to capturing $T C^{0}$, too. We thus obtain

$$
\operatorname{FOC}(<,+) \subsetneq \operatorname{FOC}(<,+, \times)=T C^{0} \stackrel{?}{\neq} \text { LOGSPACE. }
$$




\subsection{Related work}

Most of the previous work on the expressibility of counting quantifiers considered two-sorted structures, which consist of a finite "vertex domain" (the 'actual' structure) and a (possibly infinite) number domain.

Infinite number domains with arbitrary operations, as introduced by Grädel and Gurevich in their 'meta-finite model theory' [11], were considered by Benedikt and Keisler [4], who proved separations for several variations of counting quantifiers on such structures.

In the work more closely related to this paper, finite structures of the form

$$
\mathcal{A}=\left(\{0,1, \ldots, N\},\left\{v_{0}, v_{1}, \ldots, v_{N}\right\},<,+, \times, R_{1}^{\mathcal{A}}, \ldots, R_{r}^{\mathcal{A}}\right)
$$

were considered. Here, the relations $R_{i}^{\mathcal{A}}$ apply only to the vertex domain $\left\{v_{0}, \ldots, v_{N}\right\}$, and the ordering and arithmetic operations apply only to the number domain $\{0,1, \ldots, N\}$. For a counting formula $\exists^{\geq} y \varphi(x), x$ ranges over the vertex domain, while $y$ ranges over the number domain.

By splitting the structure in this way, it is possible to consider structures that are not ordered. Etessami [8] showed that if one has only a successor relation $S$ on the vertex domain, then first-order logic with counting, addition and multiplication cannot express the transitive closure of $S$, and therefore fails to capture LOGSPACE. The proof in the journal version of the paper relies on the fact that first-order logic with counting quantifiers can only express local properties, which follows from [22]. Libkin [18, 19, 20] also considered local properties of a variety of logics involving counting quantifiers.

Unfortunately, in the presence of a total ordering, these proof techniques do not apply anymore, since all elements of the structure are directly "connected" by the ordering.

The result of this paper may therefore be one of the very few non-trivial statements about the expressibility of firstorder logic with counting quantifiers on ordered structures. By avoiding two-sorted structures, it also seems more natural than other results in this area.

To prove our main result, we give an explicit winning strategy for player II in an Ehrenfeucht-Fraïssé-game for the logic FOC. Winning strategies for Ehrenfeucht-Fraïsségames for first order logic without counting quantifiers, but in the presence of an addition predicate, have been studied before. Most of them are related to techniques used by Presburger for the quantifier elimination of $T h(\mathbb{Z})[23]$; a very readable exposition of such a strategy can be found in section 3.3 of [12]. Lynch [21] also gave a similar strategy when proving inexpressibility results for first-order logic on structures with an addition relation.

The main technical contribution of this paper is the extension of the strategy in the presence of counting quan- tifiers, corresponding to a 'set move' in the EhrenfeuchtFraïssé-game.

After completing this paper, we noticed a reference in [8] to an (as yet) unpublished manuscript of Lindell that seems to imply the result stated in this paper. Our result was derived independently, and might also be interesting in its own right due to its proof technique.

\subsection{Outline of paper}

This paper is structured as follows. In section 2, we present the notions and definitions from finite model theory used in this paper. In section 3 we state and prove our main theorem. In the last section 4 we summarize our results and discuss possible future research directions.

\section{Logics and games}

In this section we give the definitions necessary for the rest of the paper, in particular of the logic $\operatorname{FOC}(<,+)$ which will be the main concern of this paper. We then describe the Ehrenfeucht-Fraïssé-game for this logic, a gametheoretic method to prove inexpressibility results. Our notation follows $[5,6]$.

\subsection{Remarks on Notation}

Our notation slightly deviates from the other literature on counting quantifiers. This is due to the fact that we consider only one-sorted structures. In the presence of a total ordering on the structure, a separate number domain does not give any additional expressibility ${ }^{1}$, so for simplicity we avoid using it.

Usually the name $F O(\mathbf{C})$ is given to first-order logic with quantifiers on two-sorted structures with the BIT predicate on the "number domain". In order not to confuse the reader, we will call first-order order logic with counting on single-sorted domains $F O C$.

\subsection{Structures}

All structures we consider in this paper are finite with domains $\{0,1, \ldots, N\}$ where $N \in \mathbb{N}$. If we consider $\sigma$ structures (where $\sigma$ is the set $\left\{R_{1}, \ldots, R_{r}\right\}$ of relations on the structure) and $\sigma$ includes one or more of the relation symbols $<,+$ and $\times$, then these relations are always interpreted as the "natural" ordering, addition and multiplication relations, respectively, on the set $\{0, \ldots, N\}$. Note that + and $\times$ are always relations, not functions, in this paper.

\footnotetext{
${ }^{1}$ One can identify each number $i$ with the unique 'vertex' element $v_{i}$ that satisfies $\exists^{=i}$ y $y<x$, and in this way use all relations of the number domain also on the vertex domain, and vice versa.
} 


\subsection{FOC}

The logic FOC studied in the following is the extension of first order logic obtained by adding counting quantifiers. These quantifiers have the following form:

$\exists^{\geq y} x \varphi$ where $x, y$ are variables and $\varphi$ is a $F O C$-formula.

The variable $x$ in $\exists^{\geq y} x \varphi$ is bound by the quantifier, while $y$ is still free. The formula is satisfied by a structure $\mathcal{A}=(A, \bar{R})$ and a variable assignment $\beta$ iff there are at least $\beta(y)$ distinct elements $a \in A$, such that $\left(\mathcal{A}, \beta \frac{a}{x}\right) \models \varphi$.

Definition $1(F O C, F O C(<,+), F O C(<,+, \times), \ldots)$ $F O C$ is the logic obtained by adding counting quantifiers to first order logic. For all $\tau \subseteq\{+, \times,<\}, F O C(\tau)$ is FOC restricted to structures that have the relations from $\tau$, interpreted in the 'natural' way.

Note that $F O C(+), F O C(<)$ and $F O C(<,+)$ have the same expressive power, since an ordering can be defined in terms of an addition, and vice versa ${ }^{2}$ :

$$
\begin{aligned}
& a<b \Longleftrightarrow \quad \exists x(a+x=b \wedge x+x \neq x), \\
& a+b=c \quad \Longleftrightarrow \quad \exists^{=b} x(a<x \wedge x \leq c) .
\end{aligned}
$$

\subsection{Capturing complexity}

Central to the relationship between finite model theory and complexity theory is the notion of a logic capturing a complexity class. Complexity classes are usually defined in terms of languages, but strings over a finite alphabet can easily be encoded as (ordered) relational structures. This means that strings and structures can be used interchangeably, which leads to the following definition.

\section{Definition 2 (Capturing a complexity class)}

$A \operatorname{logic} L$ is said to capture a complexity class $C$ iff for every class $K$ of $\sigma$-structures in $C$ there is a sentence $\varphi \in L$ (and vice versa), such that for all $\sigma$-structures $\mathcal{A}$ the following holds:

$$
\mathcal{A} \in K \quad \Longleftrightarrow \mathcal{A} \models \varphi
$$

( $\mathcal{A}$ is in $K$ iff $\mathcal{A}$ satisfies the sentence $\varphi$ ).

Following Fagin's 1974 result [9] that the logic $\Sigma_{1}^{1}$ (existential second order logic) captures $N P$, logical characterizations have been found for many other complexity classes such as $P$ [13, 24], PSPACE [1], and LOGSPACE [14]. For the latter characterizations the structures have to be ordered, as otherwise the logics fail to express even simple statements such as 'the structure has an even size'. It is an

\footnotetext{
${ }^{2}$ Here $\exists^{=b} x \varphi$ is used as shorthand for $\exists^{\geq} x \varphi \wedge \forall y\left(\exists^{\geq} x \varphi \rightarrow y \leq b\right)$. We also write $x+y=z$ instead of $+x y z$.
}

open problem whether there exist logical characterizations of these classes that also hold for structures without an ordering.

As mentioned in the introduction, Barrington et al. showed the following theorem in [3].

\section{Theorem 1 (Barrington, Immerman, Straubing)} FOC $(<,+, \times)$ captures $T C^{0}$.

To be precise, instead of + and $\times$, Barrington et al. use a predicate called $B I T$, where $B I T(i, j)$ is true iff the $i$-th bit in the binary representation of $j$ is 1 . But $B I T$ and $\{+, \times\}$ have the same expressive power when added to first-order logic with counting quantifiers (for a proof see, e.g, section 1.2.1 in [17]).

\subsection{Ehrenfeucht-Fraïssé Games}

Ehrenfeucht-Fraïssé games (EF-games) are a gametheoretic method to prove inexpressibility results for $\log$ ics. They were invented by Ehrenfeucht [7] based on work by Fraïsse [10]. We will give a short introduction to EFgames, and refer the reader to [5] for a more thorough coverage. Whenever we use the term "EF-game" in this paper, we mean an EF-game for the logic FOC.

An EF-game proceeds as follows. Given are two structures $\mathcal{A}$ and $\mathcal{B}$ (with domains $A$ and $B$, respectively) of the same signature and a number $M \geq 1$. The game consists of $M$ moves, where two players I and II (also called "spoiler" and "duplicator", respectively) choose elements $\left(a_{i}\right)_{i=1, \ldots, M}$ from $\mathcal{A}$ and elements $\left(b_{i}\right)_{i=1, \ldots, M}$ from $\mathcal{B}$, according to the following rules.

Every move starts by player I deciding whether to make a point move or a set move. Then the following happens (assume this is the $m^{\text {th }}$ move and $a_{1}, b_{1}, \ldots, a_{m-1}, b_{m-1}$ have already been chosen).

\section{Point Move:}

1. Player I chooses a structure $(\mathcal{A}$ or $\mathcal{B})$. If I chooses $\mathcal{A}$, I picks an element $a_{m}$ from that structure, otherwise, I picks an element $b_{m}$ from $\mathcal{B}$.

2. Player II then selects an element from the other structure, so if I chooses $\mathcal{A}$, II picks an element $b_{m}$ from $\mathcal{B}$, and if I chose $\mathcal{B}$, II picks an element $a_{m}$ from $\mathcal{A}$.

\section{Set Move:}

1. Player I chooses a structure $(\mathcal{A}$ or $\mathcal{B})$ - for the following we assume I chose $\mathcal{A}$ (otherwise exchange the roles of $\mathcal{A}$ and $\mathcal{B}$ ). Player I then picks an $i<m$ and a set $A_{0} \subseteq A$ with $\left|A_{0}\right|=a_{i}$.

2. Player II picks a subset $B_{0} \subseteq B$ with $\left|B_{0}\right|=b_{i}$. 
3. Player I chooses an element $b_{m}$ from $B_{0}$.

4. Player II selects an element $a_{m}$ from $A_{0}$.

Note that if we have $a_{i}=b_{i}=1$ for some $i$, then the point move is just a special case of the set move. We nevertheless make the distinction, since it helps structure our reasoning later on; we can first consider the 'simple' point move, and then extend our results to the more complex set move.

Player II wins a $M$-Move EF-game iff after $M$ moves the mapping $\left(a_{1}, a_{2}, \ldots, a_{M}\right) \mapsto\left(b_{1}, b_{2}, \ldots, b_{M}\right)$ is a partial isomorphism from $\mathcal{A}$ to $\mathcal{B}$, i.e. it preserves equality and the relations on the two structures. Otherwise, player I wins.

Obviously, if $\mathcal{A}$ and $\mathcal{B}$ are isomorphic, player II can always win by mirroring the moves of player $\mathrm{I}$ in the other structure. But more generally the following theorem holds - a variant of the theorem due to Ehrenfeucht for first-order logic. A proof can be found in [8].

\section{Theorem 2}

The following two statements are equivalent:

(i) Player II has a winning strategy for the $M$-move EFgame on two structures $\mathcal{A}$ and $\mathcal{B}$.

(ii) $\mathcal{A}$ and $\mathcal{B}$ satisfy the same $F O C$ formulas of quantifier rank $\leq M$.

For inexpressibility results we use the following corollary of Theorem 2:

\section{Corollary 3}

Let $K$ and $L$ be classes of finite structures, such that there exist sequences $\left(\mathcal{A}_{i}\right)_{i \in \mathbb{N}}$ in $K$ and $\left(\mathcal{B}_{i}\right)_{i \in \mathbb{N}}$ in $L$, where for every $M \geq 1$ player $I I$ has a winning strategy for the $M$ move EF-game on $\mathcal{A}_{M}$ and $\mathcal{B}_{M}$.

Then $K$ and $L$ cannot be distinguished by a FOCformula.

\section{Main Theorem}

"Deterministic transitive closure" is the following question: Given a directed graph $G=(V, E)$, where every node has out-degree at most 1 , and two nodes $s, t \in V$; is there a path in $G$ from $s$ to $t$ ? This problem is obviously in LOGSPACE. Our main theorem, which we prove in this section, is the following.

\section{Theorem 4}

Deterministic transitive closure (DTC) cannot be expressed in $\operatorname{FOC}(<,+)$.

It then immediately follows that

\section{Corollary 5}

FOC $(<,+)$ does not capture LOGSPACE.

\subsection{Proof of main theorem}

For each natural number $M$ we construct two structures $\mathcal{A}_{M}=\left(\left\{0, \ldots, N_{M}\right\},<,+, E^{\mathcal{A}_{M}}\right)$ and $\mathcal{B}_{M}=\left(\left\{0, \ldots, N_{M}\right\}\right.$, $\left.<,+, E^{\mathcal{B}_{M}}\right)$, where $E$ is a binary relation that represents a directed graph where every vertex has an out-degree of at most 1 . We will have that

(i) 0 and $N_{M}$ are connected via an $E^{\mathcal{A}_{M}}$-path

(ii) 0 and $N_{M}$ are not connected via an $E^{\mathcal{B}_{M}}$-path

(iii) Player II has a winning strategy for the $M$-move Ehrenfeucht-Fraïssé Game on $\mathcal{A}_{M}$ and $\mathcal{B}_{M}$.

Since the first and last element of the ordering, 0 and $N_{M}$, are $F O$-definable in ordered structures, Corollary 3 then immediately implies that $D T C$ cannot be expressed in $\operatorname{FOC}(<,+)$.

So let $M \geq 1$ be fixed for the rest of this proof, and we just write $\mathcal{A}, \mathcal{B}, N$ instead of $\mathcal{A}_{M}, \mathcal{B}_{M}, N_{M}$.

\subsection{Construction of $\mathcal{A}$ and $\mathcal{B}$}

To define the size of the structures $\mathcal{A}$ and $\mathcal{B}$, we use a function $r:\{4, \ldots, M+4\} \rightarrow \mathbb{N}$ that will also play a role later in the proof. During the proof we will repeatedly make use of the fact that $r(m)$ is 'much larger' than $r(m+1)$ for all $m \in\{4, \ldots, M+3\}$. In fact, the exact definition of $r$ does not matter much, as long as it satisfies all size constraints imposed by the proof. When reading the proof, it is therefore helpful to ignore the exact definition of $r$, and just convince oneself that all constraints in the proof can be satisfied simultaneously.

The function $r$ is recursively defined as follows:

- $r(M+4):=1$

- $r(m):=\left((6 r(m+1))^{12 m} \cdot \alpha\right)^{\alpha}$ where $\alpha=\operatorname{lcm}(1,2, \ldots, r(m+1))+2$.

Set $R:=3 r(4)^{3} \cdot r(4)$ ! and fix the size of our structures as $N:=(4 R+1) R$.

We define the edge relations as follows:

$$
\begin{aligned}
& E^{\mathcal{A}}:=\{(a, b) \mid a+R=b\} \\
& E^{\mathcal{B}}:=\{(a, b) \mid a+2 R=b\}
\end{aligned}
$$

Obviously 0 and $N$ are connected via an $E^{\mathcal{A}}$-path (since $N$ is a multiple of $R$ ) but not via an $E^{\mathcal{B}}$-path (since $N$ is not a multiple of $2 R$ ). 


\subsection{Strategy}

We have to show that these two structures cannot be distinguished using an $M$-move Ehrenfeucht-Fraïssé-game. To this end we state a strategy for player II that ensures that all mappings $\left(a_{1}, \ldots, a_{m}\right) \mapsto\left(b_{1}, \ldots, b_{m}\right)$ occurring during the game will be partial isomorphisms.

The crucial strategy element for player II is to make certain that all partial isomorphisms $\left(a_{1}, \ldots, a_{m}\right) \mapsto$ $\left(b_{1}, \ldots, b_{m}\right)$ occurring during the game can be extended to linear combinations $\sum \alpha_{i} a_{i} \mapsto \sum \alpha_{i} b_{i}$ with "simple" coefficients $\alpha_{i}$. If this were not the case, player I could quickly establish that the two structures are not isomorphic.

For example, if we have $a_{2}=5 \cdot a_{1}$ in one structure, but $b_{2} \neq 5 \cdot b_{1}$ in the other structure, then player I could play $2 \cdot a_{1}, 3 \cdot a_{1}, 5 \cdot a_{1}$ in $\mathcal{A}$, and player II would be forced to respond with $2 \cdot b_{1}, 3 \cdot b_{1}, 5 \cdot b_{1}$ since the new elements can be expressed as sums of already chosen elements. Apparently player II would lose in this case.

More precisely, the linear combinations player II must preserve have the following form.

Definition $3\left(L_{d}(\bar{a})\right)$

$$
\begin{aligned}
& L_{d}\left(a_{1}, \ldots, a_{m}\right):= \\
& \left\{\sum_{i=1}^{m} \frac{p_{i}}{q_{i}} \cdot a_{i} \in \mathbb{Z}\left|p_{i}, q_{i} \in \mathbb{Z},\right| p_{i}|,| q_{i} \mid \leq d, q_{i} \neq 0\right\}
\end{aligned}
$$

We can now define sets $W_{m}(4 \leq m \leq M+4)$ that are winning situations for player II. The function $r$ used in the definition is the one we used above to define the size $N$ of our structures. Note that $r$ decreases rapidly; intuitively, at the beginning of the game, player II has to preserve a large number of linear equations to account for all possible strategies player I might take, while as the game progresses, player II has to preserve fewer and fewer linear combinations.

\section{Definition $4\left(W_{m}\right)$}

$$
\begin{aligned}
W_{m}:=\{ & \left(a_{1}, \ldots, a_{m}\right) \mapsto\left(b_{1}, \ldots, b_{m}\right) \mid \\
& \left(a_{1}, a_{2}, a_{3}, a_{4}\right)=(0,1, R, N), \\
& \left(b_{1}, b_{2}, b_{3}, b_{4}\right)=(0,1,2 R, N), \\
& \pi: L_{r(m)}\left(a_{1}, \ldots, a_{m}\right) \rightarrow L_{r(m)}\left(b_{1}, \ldots, b_{m}\right) \text { with } \\
& \pi\left(\sum_{i=1}^{m} \frac{p_{i}}{q_{i}} a_{i}\right)=\sum_{i=1}^{m} \frac{p_{i}}{q_{i}} b_{i} \\
& \text { is a (well-defined) isomorphism }\}
\end{aligned}
$$

"Well-defined isomorphism" means that

$$
\sum_{i=1}^{m} \frac{p_{i}}{q_{i}} a_{i} \in \mathbb{Z} \Longleftrightarrow \sum_{i=1}^{m} \frac{p_{i}}{q_{i}} b_{i} \in \mathbb{Z}
$$

and

$$
\sum_{i=1}^{m} \frac{p_{i}}{q_{i}} a_{i}=\sum_{i=1}^{m} \frac{p_{i}^{\prime}}{q_{i}^{\prime}} a_{i} \Longleftrightarrow \sum_{i=1}^{m} \frac{p_{i}}{q_{i}} b_{i}=\sum_{i=1}^{m} \frac{p_{i}^{\prime}}{q_{i}^{\prime}} b_{i}
$$

for all $p_{i}, p_{i}^{\prime}, q_{i}, q_{i}^{\prime}$ with absolute value $\leq r(m)$, and that $\pi$ is an isomorphism preserving $<,+$ and $E$.

Note that for every mapping $\left(a_{1}, \ldots, a_{m}\right) \mapsto$ $\left(b_{1}, \ldots, b_{m}\right) \in W_{m}$ we must then necessarily have $a_{i} \equiv b_{i} \bmod \operatorname{lcm}(1,2, \ldots, r(m))$ for all $i \in\{1, \ldots, m\}$, since otherwise $\pi$ would not preserve integrality.

Our claim that player II has a winning strategy for the $M$ move EF-game can be split into the following two lemmas.

\section{Lemma 6}

$(0,1, R, N) \mapsto(0,1,2 R, N) \in W_{4}$ (i.e. $W_{4}$ is not empty)

\section{Lemma 7}

If a game-situation $\left(a_{1}, \ldots, a_{m}\right) \mapsto\left(b_{1}, \ldots, b_{m}\right) \in W_{m}(m<$ $M+4)$ arises, player II can always enforce that $a_{m+1}$ and $b_{m+1}$ are chosen such that $\left(a_{1}, \ldots, a_{m+1}\right) \mapsto\left(b_{1}, \ldots, b_{m+1}\right)$ is in $W_{m+1}$.

I.e., we actually show a stronger statement than player II just having a winning strategy for the $M$-move EF-game. We show that II can win a $M$-move game, where four elements in each structure $((0,1, R, N)$ and $(0,1,2 R, N))$ are already fixed at the beginning of the game.

Proof of Lemma 6: We have to show that the mapping $(0,1, R, N) \mapsto(0,1,2 R, N)$ can be extended to an isomorphism $\pi: L_{r(4)}(0,1, R, N) \rightarrow L_{r(4)}(0,1,2 R, N)$. Intuitively, the reason for this being possible is that the elements of the two quadruples belong to the same residue classes for all moduli less than $r(4)$, and that $1,2 R$ and $N$ are so 'far apart' that they satisfy only trivial addition relations.

More formally, first, we have that for all $p_{i}, q_{i}(1 \leq i \leq 3)$ of no more than $r(4)$ in absolute value, the following holds.

$$
\frac{p_{1}}{q_{1}} \cdot 1+\frac{p_{2}}{q_{2}} \cdot R+\frac{p_{3}}{q_{3}} \cdot N \in \mathbb{Z} \Longleftrightarrow \frac{p_{1}}{q_{1}} \cdot 1+\frac{p_{2}}{q_{2}} \cdot 2 R+\frac{p_{3}}{q_{3}} \cdot N \in \mathbb{Z}
$$

To see this, just note that $R, 2 R$ and $N$ are multiples of $r(4)$ !, and therefore $q_{2}$ and $q_{3}$ evenly divide $R, 2 R$, and $N$. Thus, both sums are integral if and only if $p_{1} / q_{1}$ is an integer.

Next, we have to check whether $\pi$ preserves equality and ordering. It suffices to show that for all $p_{i}, q_{i}(1 \leq i \leq 6)$ of 
no more than $r(4)$ in absolute value, the following holds.

$$
\begin{aligned}
& \operatorname{sign}\left(\frac{p_{1}}{q_{1}} \cdot 1+\frac{p_{2}}{q_{2}} \cdot R+\frac{p_{3}}{q_{3}} \cdot N-\left(\frac{p_{4}}{q_{4}} \cdot 1+\frac{p_{5}}{q_{5}} \cdot R+\frac{p_{6}}{q_{6}} \cdot N\right)\right) \\
= & \operatorname{sign}\left(\frac{p_{1}}{q_{1}} \cdot 1+\frac{p_{2}}{q_{2}} \cdot 2 R+\frac{p_{3}}{q_{3}} \cdot N-\left(\frac{p_{4}}{q_{4}} \cdot 1+\frac{p_{5}}{q_{5}} \cdot 2 R+\frac{p_{6}}{q_{6}} \cdot N\right)\right)
\end{aligned}
$$

To see that this equality holds, note that $N$ was chosen such that it is much larger than $R$, and $R$ is much larger than 1. If $\frac{p_{3}}{q_{3}} \neq \frac{p_{6}}{q_{6}}$ we have

$$
\begin{aligned}
\left|\frac{p_{1}}{q_{1}}\right| & +\left|\frac{p_{4}}{q_{4}}\right|+\left(\left|\frac{p_{2}}{q_{2}}\right|+\left|\frac{p_{5}}{q_{5}}\right|\right) \cdot 2 R \\
& \leq 8 r(4) \cdot R<\frac{N}{r(4)^{2}}<\left|\frac{p_{3}}{q_{3}}-\frac{p_{6}}{q_{6}}\right| \cdot N
\end{aligned}
$$

Thus, the values of expressions (1) and (2) are both equal to the sign of $\frac{p_{3}}{q_{3}}-\frac{p_{6}}{q_{6}}$.

If, on the other hand, $\frac{p_{3}}{q_{3}}=\frac{p_{6}}{q_{6}}$ holds, but $\frac{p_{2}}{q_{2}} \neq \frac{p_{5}}{q_{5}}$, then we have

$$
\left|\frac{p_{1}}{q_{1}}\right|+\left|\frac{p_{4}}{q_{4}}\right| \leq 2 r(4) \leq \frac{R}{r(4)^{2}}<\left|\frac{p_{2}}{q_{2}}-\frac{p_{5}}{q_{5}}\right| \cdot R
$$

and the expressions (1) and (2) are both equal to the sign of $\frac{p_{2}}{q_{2}}-\frac{p_{5}}{q_{5}}$. In the case where $\frac{p_{2}}{q_{2}}=\frac{p_{5}}{q_{5}}$ and $\frac{p_{3}}{q_{3}}=\frac{p_{6}}{q_{6}}$, the expressions are identical.

Checking that $\pi$ respects + can be done in a similar fashion; a little calculation shows that

$$
\frac{p_{1}}{q_{1}}+\frac{p_{2}}{q_{2}} R+\frac{p_{3}}{q_{3}} N+\frac{p_{1}^{\prime}}{q_{1}^{\prime}}+\frac{p_{2}^{\prime}}{q_{2}^{\prime}} R+\frac{p_{3}^{\prime}}{q_{3}^{\prime}} N=\frac{p_{1}^{\prime \prime}}{q_{1}^{\prime \prime}}+\frac{p_{2}^{\prime \prime}}{q_{2}^{\prime \prime}} R+\frac{p_{3}^{\prime \prime}}{q_{3}^{\prime \prime}} N
$$

holds if and only if for all $i \in\{1,2,3\}$ :

$$
\frac{p_{i}}{q_{i}}+\frac{p_{i}^{\prime}}{q_{i}^{\prime}}=\frac{p_{i}^{\prime \prime}}{q_{i}^{\prime \prime}}
$$

And the same is true if we replace $R$ by $2 R$. So these relations are trivially preserved by $\pi$.

The mapping $\pi$ then also respects $E$, since the edgerelation is defined in terms of the addition relation and the fixed elements $R$ and $2 R$.

Proof of Lemma 7: We will show this lemma by stating an explicit strategy for player II. First we consider the easier point move case. As mentioned in the introduction, strategies for this case can also be found in $[12,21]$. Then we will state the set move strategy which will be a non-trivial generalization of the point move.

Assume that the game situation $\left(a_{1}, \ldots, a_{m}\right) \mapsto$ $\left(b_{1}, \ldots, b_{m}\right) \in W_{m}$ has arisen, where $\left(a_{1}, \ldots, a_{4}\right)=$ $(0,1, R, N)$ and $\left(b_{1}, \ldots, b_{4}\right)=(0,1,2 R, N)$. Let $\pi$ be the induced isomorphism between $L_{r(m)}\left(a_{1}, \ldots, a_{m}\right)$ and $L_{r(m)}\left(b_{1}, \ldots, b_{m}\right)$. In the following we will abbreviate the tuples $\left(a_{1}, \ldots, a_{m}\right)$ and $\left(b_{1}, \ldots, b_{m}\right)$ as $\bar{a}$ and $\bar{b}$, respectively.

\section{Point Move}

Assume that player I chooses a new element $a_{m+1}$ in structure $\mathcal{A}$ (the case of player I choosing an element of $\mathcal{B}$ is handled symmetrically). We have to choose a new element $b_{m+1}$ such that $\left(\bar{a}, a_{m+1}\right) \mapsto\left(\bar{b}, b_{m+1}\right)$ is in $W_{m+1}$. We distinguish two cases.

In the first case, $a_{m+1}$ is "close" to the previously chosen elements, and already in the domain of $\pi$. Here we can just set $b_{m+1}:=\pi\left(a_{m+1}\right)$. In the second case, $a_{m+1}$ is "far" from all previously chosen elements, i.e. does not satisfy any non-trivial addition relations with them. In this case it suffices for $b_{m+1}$ to satisfy the same ordering constraints as $a_{m+1}$ and belong to the same residue classes as $a_{m+1}$ for moduli less than $r(m+1)$. We will see that this is always possible, since $r(m)$ was chosen large enough to leave plenty of 'room' between the elements $\left\{b_{i}\right\}$.

We will now give the details of the strategy in the two cases.

Case 1: $a_{m+1} \in L_{3 r(m+1)^{6}}(\bar{a})$

Here, $a_{m+1}$ is a linear combination $a_{m+1}=\sum_{i=1}^{m} \frac{p_{i}^{\prime}}{q_{i}^{\prime}} a_{i}$ of $\left\{a_{1}, \ldots, a_{m}\right\}$ with "small" coefficients. Therefore any linear combination $\sum_{i=1}^{m+1} \frac{p_{i}}{q_{i}} a_{i}$ of $\left\{a_{1}, \ldots, a_{m+1}\right\}$ can be rewritten as a linear combination

$$
\sum_{i=1}^{m}\left(\frac{p_{i}}{q_{i}}+\frac{p_{m+1} p_{i}^{\prime}}{q_{m+1} q_{i}^{\prime}}\right) a_{i}
$$

of $\left\{a_{1}, \ldots, a_{m}\right\}$ with "slightly larger" coefficients. So we have

$$
L_{r(m+1)}\left(\bar{a}, a_{m+1}\right) \subseteq L_{6 r(m+1)^{8}}(\bar{a}) \subseteq L_{r(m)}(\bar{a}),
$$

where the last inclusion holds by definition of $r(m)$. Thus, by setting $b_{m+1}:=\pi\left(a_{m+1}\right)$ we have an isomorphism between $L_{r(m+1)}\left(\bar{a}, a_{m+1}\right)$ and $L_{r(m+1)}\left(\bar{b}, b_{m+1}\right)$ that is just a restriction of the old isomorphism $\pi$.

Case 2: $a_{m+1} \notin L_{3 r(m+1)^{6}}(\bar{a})$

Let $a_{\min }$ and $a_{\max }$ be the greatest (respectively least) elements of $L_{3 r(m+1)^{6}}(\bar{a})$ less (respectively greater) than $a_{m+1}$, and $b_{\min }$ and $b_{\max }$ be their images under $\pi$.

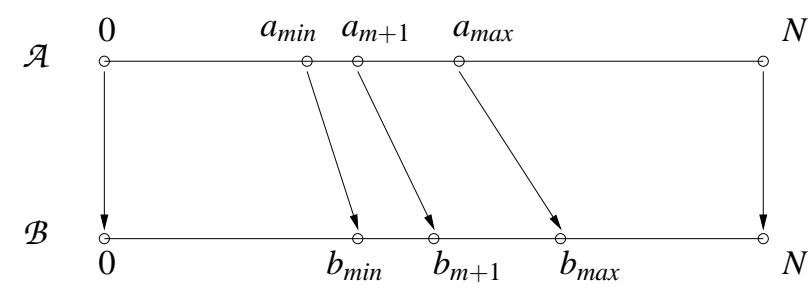

We claim that it is sufficient for player II to choose $b_{m+1}$ such that 
- $b_{m+1} \in\left\{b_{\min }+1, \ldots, b_{\max }-1\right\}$, and

- $a_{m+1} \equiv b_{m+1} \bmod \operatorname{lcm}(1,2, \ldots, r(m+1))$

Let us first see that it is always possible to find such a $b_{m+1}$. If $b_{\max }-b_{\min }>\operatorname{lcm}(1, \ldots, r(m+1))$, then such a $b_{m+1}$ obviously exists, since there has to be an element belonging to the same residue class as $a_{m+1}$ between $b_{\min }$ and $b_{\max }$.

So assume that $b_{\max }-b_{\min } \leq \operatorname{lcm}(1, \ldots, r(m+1))$ holds. Since, by definition, $r(m) \geq \operatorname{lcm}(1,2, \ldots, r(m+1))$ we have $\{1, \ldots, \operatorname{lcm}(1,2, \ldots, r(m+1))\} \subseteq L_{r(m)}(\bar{a})$ (as "linear combinations" of $\left.a_{2}=b_{2}=1\right)$. Since $\pi$ is the identity mapping on these linear combinations of 1 and preserves the addition predicate, we have that $a_{\max }-a_{\min }=b_{\max }-b_{\min }$. Furthermore we have for all $k \leq \operatorname{lcm}(1,2, \ldots, r(m+1))$ that

$$
\frac{a_{\max }+k}{\operatorname{lcm}(1,2, \ldots, r(m+1))} \in \mathbb{Z} \Leftrightarrow \frac{b_{\max }+k}{\operatorname{lcm}(1,2, \ldots, r(m+1))} \in \mathbb{Z}
$$

and therefore $a_{\max } \equiv b_{\max } \bmod \operatorname{lcm}(1,2, \ldots, r(m+1))$, and the same for $a_{\min }$ and $b_{\min }$.

So $a_{\min }$ and $b_{\min }$ (and $a_{\max }$ and $b_{\max }$ ) belong to the same residue classes modulo $\operatorname{lcm}(1,2, \ldots, r(m+1))$. It then follows with $a_{\max }-a_{\min }=b_{\max }-b_{\min }$ that for each residue class $a_{m+1}$ modulo $\operatorname{lcm}(1,2, \ldots, r(m+1))$ between $a_{\min }$ and $a_{\max }$ the same residue class exists between $b_{\min }$ and $b_{\max }$, and we can choose a $b_{m+1}$ according to the restrictions given above.

After having seen that we can always choose a $b_{m+1}$ according to the restrictions given above, we now have to show that this choice of $b_{m+1}$ necessarily leads to a mapping $\left(\bar{a}, a_{m+1}\right) \mapsto\left(\bar{b}, b_{m+1}\right) \in W_{m+1}$.

So let $b_{m+1}$ be chosen as above and let $\pi^{\prime}$ : $L_{r(m+1)}\left(\bar{a}, a_{m+1}\right) \rightarrow L_{r(m+1)}\left(\bar{b}, b_{m+1}\right)$ be the extension of the mapping $\left(\bar{a}, a_{m+1}\right) \mapsto\left(\bar{b}, b_{m+1}\right)$. We have to show that $\pi^{\prime}$ is a well-defined isomorphism, which requires checking the same properties as in the proof of Lemma 6 .

- First, we have that

$$
\sum_{i=1}^{m+1} \frac{p_{i}}{q_{i}} a_{i} \in \mathbb{Z} \Longleftrightarrow \sum_{i=1}^{m+1} \frac{p_{i}}{q_{i}} b_{i} \in \mathbb{Z}
$$

since for every $i$ both $a_{i}$ and $b_{i}$ lie in the same residue class $^{3}$ modulo $1 \mathrm{~cm}(1, \ldots, r(m+1))$ and therefore modulo $\operatorname{lcm}\left(q_{1}, q_{2}, \ldots, q_{m+1}\right)$.

- $\pi^{\prime}$ respects equality and ordering. Let $a=$ $\sum_{i=1}^{m+1} \frac{p_{i}}{q_{i}} a_{i}$ and $a^{\prime}=\sum_{i=1}^{m+1} \frac{p_{i}^{\prime}}{q_{i}^{\prime}} a_{i}$ be two elements of

\footnotetext{
${ }^{3}$ For $a_{m+1}$ and $b_{m+1}$ we have this by choice of $b_{m+1}$, for the other $i$ this holds since $\pi$ preserves integrality, i.e. since we have $\frac{p+a_{i}}{q} \in \mathbb{Z} \Leftrightarrow \frac{p+b_{i}}{q} \in \mathbb{Z}$ for all $p, q \leq r(m+1)$.
}

$L_{r(m+1)}\left(\bar{a}, a_{m+1}\right)$ with $a<a^{\prime}$ (the case $a=a^{\prime}$ is handled in the same manner). If $\frac{p_{m+1}}{q_{m+1}}=\frac{p_{m+1}^{\prime}}{q_{m+1}^{\prime}}$ then the inequality is equivalent to

$$
\sum_{i=1}^{m} \frac{p_{i}}{q_{i}} a_{i}<\sum_{i=1}^{m} \frac{p_{i}^{\prime}}{q_{i}^{\prime}} a_{i}
$$

This inequality is independent of $a_{m+1}$, so since $\pi^{\prime}$ agrees with $\pi$ on $L_{r(m+1)}(\bar{a})$ and $\pi$ respects the ordering, we have that

$$
\sum_{i=1}^{m} \frac{p_{i}}{q_{i}} b_{i}<\sum_{i=1}^{m} \frac{p_{i}^{\prime}}{q_{i}^{\prime}} b_{i}
$$

And adding $\frac{p_{m+1}}{q_{m+1}} b_{m+1}=\frac{p_{m+1}^{\prime}}{q_{m+1}^{\prime}} b_{m+1}$ on both sides of the inequality does not change its validity.

If, on the other hand $\frac{p_{m+1}}{q_{m+1}} \neq \frac{p_{m+1}^{\prime}}{q_{m+1}^{\prime}}$ (say it is $>$ ), then the inequality can be solved for $a_{m+1}$ :

$$
a_{m+1}<\sum_{i=1}^{m}\left(\frac{q_{m+1} q_{m+1}^{\prime}}{p_{m+1} q_{m+1}^{\prime}-p_{m+1}^{\prime} q_{m+1}}\right)\left(\frac{p_{i}^{\prime} q_{i}-p_{i} q_{i}^{\prime}}{q_{i} q_{i}^{\prime}}\right) a_{i}
$$

The linear combination on the right is an element of $L_{2 r(m+1)^{4}}(\bar{a})$, and therefore an element of $L_{3 r(m+1)^{6}}(\bar{a})$. Since we chose $b_{m+1}$ such that it satisfies the same ordering-relations as $a_{m+1}$ regarding the elements of $L_{3 r(m+1)^{6}}(\bar{b})$, this inequality is preserved by $\pi^{\prime}$.

- To see that $\pi^{\prime}$ preserves + , consider an equality of the following form:

$$
\sum_{i=1}^{m} \frac{p_{i}}{q_{i}} a_{i}+\sum_{i=1}^{m} \frac{p_{i}^{\prime}}{q_{i}^{\prime}} a_{i}=\sum_{i=1}^{m} \frac{p_{i}^{\prime \prime}}{q_{i}^{\prime \prime}} a_{i}
$$

If $\frac{p_{m+1}}{q_{m+1}}+\frac{p_{m+1}^{\prime}}{q_{m+1}^{\prime}}=\frac{p_{m+1}^{\prime \prime}}{q_{m+1}^{\prime \prime}}$, then the equality is independent of the value of $a_{m+1}$, and thus preserved by $\pi^{\prime}$. If it depends on the value of $a_{m+1}$, we can solve the equation for $a_{m+1}$, obtaining

$$
a_{m+1}=\sum_{i=1}^{m} \frac{\frac{p_{i}}{q_{i}}+\frac{p_{i}^{\prime}}{q_{i}^{\prime}}-\frac{p_{i}^{\prime \prime}}{q_{i}^{\prime \prime}}}{\frac{p_{m+1}^{\prime \prime}}{q_{m+1}^{\prime \prime}}-\frac{p_{m+1}}{q_{m+1}}-\frac{p_{m+1}^{\prime}}{q_{m+1}^{\prime}}} \cdot a_{i}
$$

But this implies $a_{m+1} \in L_{3 r(m+1)^{6}}(\bar{a})$, in contradiction to the choice of $a_{m+1}$.

- And finally, $E$ is preserved, since it is defined in terms of the addition predicate.

\section{Set Move}

Player I chooses an element $a_{i}$ and a set $A_{0} \subseteq\{0,1, \ldots, N\}$ with $\left|A_{0}\right|=a_{i}$ (again the case of player I choosing an element and subset from $\mathcal{B}$ is handled symmetrically). Player 
II has to answer with a set $B_{0}$ with $\left|B_{0}\right|=b_{i}$. We want to choose $B_{0}$ in such a way that whatever element player I chooses in it, we can answer according to our point move strategy with an element of $A_{0}$.

In the point move case we distinguished between two cases for a new element: whether it is in the set $L_{3 r(m+1)^{6}}(\bar{a})$ or not. Since we want to eventually apply our point move strategy, we do the same here. Let $A_{1}:=A_{0} \cap L_{3 r(m+1)^{6}}(\bar{a})$ and $A_{2}:=A_{0} \backslash A_{1}$. We will choose $B_{1}$ and $B_{2}$ such that should player I choose an element $b_{m+1}$ from $B_{1}$, we can answer (according to our point move strategy) with an element $a_{m+1}$ of $A_{1}$ and likewise for $B_{2}$ and $A_{2}$.

- Set $B_{1}:=\left\{\pi(a) \mid a \in A_{1}\right\}$, the images of $A_{1}$ under $\pi$. Clearly, if player I chooses any element in $B_{1}$ we can answer with its pre-image under $\pi$ from $A_{1}$; this is case 1 of the point move.

- For the definition of $B_{2}$, recall case 2 in the point move. We found that a $b_{m+1}$ could be answered by any $a_{m+1}$ that is in the same residue class modulo $\operatorname{lcm}(1,2, \ldots, r(m+1))$ and in the same 'interval' between elements of $L_{3 r(m+1)^{6}}(\bar{a})$ and $L_{3 r(m+1)^{6}}(\bar{b})$, respectively.

For $c, d \in L_{3 r(m+1)^{6}}(\bar{a})$, such that $c<d$ and there are no further elements of $L_{3 r(m+1)^{6}}(\bar{a})$ between $c$ and $d$, and $r \in\{0,1, \ldots, \operatorname{lcm}(1,2, \ldots, r(m+1))-1\}$, let $I_{c, d, r}$ and $I_{c, d, r}^{\prime}$ be the following sets:

$I_{c, d, r}:=\{a \mid c<a<d, a \equiv r \bmod \operatorname{lcm}(1,2, \ldots, r(m+1))\}$

$I_{c, d, r}^{\prime}:=\{b \mid \pi(c)<b<\pi(d)$,

$$
b \equiv r \bmod \operatorname{lcm}(1,2, \ldots, r(m+1))\}
$$

When player I chooses an element in $I_{c, d, r}^{\prime}$ we can answer with any element from $I_{c, d, r}$. So let $n$ be minimal such that for appropriately chosen $c_{j}, d_{j}, r_{j}$

$$
A_{2} \subseteq \bigcup_{j=1}^{n} I_{c_{j}, d_{j}, r_{j}}
$$

We then set

$$
B_{2}:=\bigcup_{j=1}^{n} I_{c_{j}, d_{j}, r_{j}}^{\prime},
$$

and as in case 2 of the point move it is clear that if player I chooses any element from $B_{2}$, say from $I_{c_{l}, d_{l}, r_{l}}^{\prime}$,

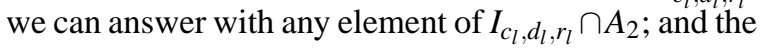
latter set is non-empty since $n$ was chosen minimally.

It follows from the previous discussion that if player II chooses $B_{0} \subseteq B_{1} \cup B_{2}$ arbitrary with $\left|B_{0}\right|=b_{i}$, then player II will always be able to answer player I's choice of $b_{m+1} \in B_{0}$ with an $a_{m+1} \in A_{0}$ according to the point move strategy. So all that remains to be shown is that $\left|B_{1} \cup B_{2}\right| \geq b_{i}$ holds, i.e., that we can actually choose a $B_{0}$ of the required size.

To this end we will show that (i) $s:=\left|L_{3 r(m+1)^{6}}(\bar{a})\right|+\sum_{i=1}^{n}\left|I_{c_{j}, d_{j}, r_{j}}\right|$ is an element of $L_{r(m)}(\bar{a})$, and that

(ii) $\pi(s)=\left|B_{1} \cup B_{2}\right|$.

Since we know that $a_{i} \leq s$, and that $\pi$ preserves the ordering, the inequality $\pi\left(a_{i}\right) \leq \pi(s)$ must also hold, i.e. we have $b_{i} \leq\left|B_{1} \cup B_{2}\right|$, and the proof is finished.

First, we bound the size of $L_{3 r(m+1)^{6}}(\bar{a})$. In the linear combinations $\sum_{i=1}^{m} \frac{p_{i}}{q_{i}} a_{i}$ of elements $a_{1}, \ldots, a_{m}$ that make up $L_{3 r(m+1)^{6}}(\bar{a})$, each of the $2 m p_{j}$ 's and $q_{j}$ 's appearing in the linear combinations can have values from $\left\{0, \pm 1, \pm 2, \ldots, \pm 3 r(m+1)^{6}\right\}$. Since $\frac{p_{j}}{q_{j}}=\frac{-p_{j}}{-q_{j}}$, etc., a lot of these choices lead to the same values. The number $\left(6 r(m+1)^{6}\right)^{2 m}$ therefore is a conservative upper bound for the number of elements in $L_{3 r(m+1)^{6}}(\bar{a})$.

This implies that $\left|L_{3 r(m+1)^{6}}(\bar{a})\right|$ is an element of $L_{\left(6 r(m+1)^{6}\right)^{2 m}}(\bar{a})$ and of $L_{\left(6 r(m+1)^{6}\right)^{2 m}}(\bar{b})$ - as a 'linear combination' of $a_{1}=b_{1}=1$.

We now turn to $\sum_{i=1}^{n}\left|I_{c_{j}, d_{j}, r_{j}}\right|$. First, the number of 'intervals' $] c_{j}, d_{j}$ [ in $L_{3 r(m+1)^{6}}(\bar{a})$ is bounded by the number of elements of that set, and therefore we have

$$
n \leq\left(6 r(m+1)^{6}\right)^{2 m} \cdot \operatorname{lcm}(1, \ldots, r(m+1)) .
$$

As for $\left|I_{c_{j}, d_{j}, r_{j}}\right|$, its size is equal to either

$$
\left\lfloor\frac{d_{j}-c_{j}-1}{\operatorname{lcm}(1,2, \ldots, r(m+1))}\right\rfloor \text { or }\left\lceil\frac{d_{j}-c_{j}-1}{\operatorname{lcm}(1,2, \ldots, r(m+1))}\right\rceil \text {. }
$$

This means that there is some

$$
e_{j} \in\{0, \pm 1, \pm 2, \ldots, \pm \operatorname{lcm}(1,2, \ldots, r(m+1))\}
$$

such that

$$
\left|I_{c_{j}, d_{j}, r_{j}}\right|=\frac{d_{j}-c_{j}-1+e_{j}}{\operatorname{lcm}(1,2, \ldots, r(m+1))} .
$$

Similarly, we have that

$$
\left|I_{c_{j}, d_{j}, r_{j}}^{\prime}\right|=\frac{\pi\left(d_{j}\right)-\pi\left(c_{j}\right)-1+e_{j}}{\operatorname{lcm}(1,2, \ldots, r(m+1))} .
$$

Using $c_{j}, d_{j} \in L_{3 r(m+1)^{6}}(\bar{a})$ this implies that

$$
\left|I_{c_{j}, d_{j}, r_{j}}\right| \in L_{\left(6 r(m+1)^{6}\right)^{2} \operatorname{lcm}(1, \ldots, r(m+1))}(\bar{a}) .
$$

And therefore

$$
\begin{aligned}
s & =\left|L_{3 r(m+1)^{6}}(\bar{a})\right|+\sum_{i=1}^{n}\left|I_{c_{j}, d_{j}, r_{j}}\right| \\
& \in L_{(n+1)\left(\left(6 r(m+1)^{6}\right)^{2 m} \operatorname{lcm}(1, \ldots, r(m+1))\right)^{(n+1)}}(\bar{a}) \subseteq L_{r(m)}(\bar{a})
\end{aligned}
$$

Here we used the (easily verified) fact, that the sum of $k$ elements of $L_{d}(\bar{a})$ is in $L_{k d^{k}}(\bar{a})$.

We also established that $\pi\left(\left|L_{3 r(m+1)^{6}}(\bar{a})\right|\right)=$ $\left|L_{3 r(m+1)^{6}}(\bar{b})\right|$, and that $\pi\left(\left|I_{c_{j}, d_{j}, r_{j}}\right|\right)=\left|I_{c_{j}, d_{j}, r_{j}}^{\prime}\right|$. Since $s$ can be expressed as the sum of these values in $L_{r(m)}(\bar{a})$, so can $\pi(s)$ in $L_{r(m)}(\bar{b})$, and therefore $\pi(s)=\left|B_{1} \cup B_{2}\right|$, as claimed. 


\section{4. $F O C(<,+)$ is weaker than $F O C(<,+, \times)$}

The previous proof gives us an immediate corollary:

\section{Corollary 8}

FOC $(<,+)$ is strictly weaker than $F O C(<,+, \times)$.

Proof: The two structures given in the preceding proof are distinguishable in $\operatorname{FOC}(<,+, \times)$ since we just have to ask whether the only element to which 0 has an edge evenly divides the maximum element.

\section{Conclusion}

We have shown that the logic $F O C(<,+)$ does not capture LOGSPACE, since it cannot express deterministic transitive closure. This may be one of the first meaningful results about the expressibility of first-order logic augmented with counting quantifiers in the presence of an ordering and non-trivial arithmetic operators.

As Corollary 8 shows, the proof breaks down in presence of a multiplication predicate. This is not surprising, as this case corresponds to the open question $T C^{0}$ vs. LOGSPACE. The intuitive reason why the problem becomes much harder is that we used the fact that the combinatorial structure of the addition operation is very simple. But, as number theory evidences, addition and multiplication taken together exhibit a very involved structure. Therefore constructing an explicit EF-game strategy for this general case seems to be very difficult.

A natural next question therefore is: if not multiplication, what other (most likely weaker) operations can we add to $F O C$, and still prove inexpressibility results?

\section{Acknowledgments}

This work was carried out while the author was at the 'Institute for Mathematical Logic' of the Albert-LudwigsUniversität in Freiburg, Germany. My thanks go to Martin Grohe, who suggested to work on this problem and gave many helpful comments on an earlier draft of this paper. I also thank Yevgeniy Dodis, Venkatesan Guruswami, Madhu Sudan and Salil Vadhan for numerous additional suggestions, and the anonymous referees for their detailed comments that helped to improve the presentation.

\section{References}

[1] S. Abiteboul and V. Vianu. Fixpoint extensions of first-order logic and datalog-like languages. Proceedings LICS'89, pages 71-79, 1989.

[2] E. Allender. Circuit complexity before the dawn of the new millennium. Proceedings FST\&TCS'96, LNCS 1180, pages 1-18, 1996.
[3] D. Barrington, N. Immerman, and H. Straubing. On uniformity within $N C^{1}$. Journal of Computer and System Sciences, 41:274-306, 1990.

[4] M. Benedikt and J. Keisler. Expressive power of unary counters. J. Mycielski (ed.), Structures in Logic and Computer Science, LNCS 1261, pages 34-50, 1997.

[5] H. Ebbinghaus and J. Flum. Finite Model Theory. Springer Verlag, 1995.

[6] H. Ebbinghaus, J. Flum, and W. Thomas. Mathematical Logic. Springer Verlag, 1994.

[7] A. Ehrenfeucht. An application of games to the completeness problem for formalized theories. Fundamenta Mathematicae, 49:129-141, 1961.

[8] K. Etessami. Counting quantifiers, successor relations and logarithmic space. Journal of Computer and System Sciences, 54:400-411, 1997.

[9] R. Fagin. Generalized first-order spectra and polynomialtime recognizable sets. R.M. Karp (ed.), Complexity of Computation, SIAM-AMS Proceedings, 7:43-73, 1974.

[10] R. Fraïsse. Sur quelques classifications des systèmes de relations. Université d'Alger, Publications Scientifiques, Série A, 1:35-182, 1954.

[11] E. Grädel and Y. Gurevich. Metafinite model theory. Information and Computation, 140:26-81, 1998.

[12] W. Hodges. Model Theory. Cambridge University Press, 1993.

[13] N. Immerman. Relational queries computable in polynomial time. Information and Control, 68:86-104, 1986.

[14] N. Immerman. Expressibility as a complexity measure: results and directions. Second Structure in Complexity Conference, pages 194-202, 1987.

[15] N. Immerman. Languages that capture complexity classes. SIAM Journal of Computing, 16(4):760-778, 1987.

[16] N. Immerman. Descriptive and computational complexity. Computational Complexity Theory, AMS Proc. Symp. in Applied Math., 38:75-91, 1989.

[17] N. Immerman. Descriptive and Computational Complexity. Springer Verlag, 1998.

[18] L. Libkin. On the forms of locality over finite models. Proceedings LICS'97, pages 204-215, 1997.

[19] L. Libkin. On counting logics and local properties. Proceedings LICS'98, pages 501-512, 1998.

[20] L. Libkin and L. Wong. Unary quantifiers, transitive closure, and relations of large degree. Proceedings STACS'98, LNCS 1373, pages 183-193, 1998.

[21] J. F. Lynch. On sets of relations definable by addition. Journal of Symbolic Logic, 47(3):659-668, 1982.

[22] J. Nurmonen. On winning strategies with unary quantifiers. Journal of Logic and Computation, 6(6):779-798, 1996.

[23] M. Presburger. Über die Vollständigkeit eines gewissen Systems der Arithmetik ganzer Zahlen, in welchem die Addition als einzige Operation hervortritt. Sprawozdanie z I Kongresu Mat. Krajów Slowiańskich, pages 92-101, 1930.

[24] M. Vardi. The complexity of relational query languages. Proceedings STOC'82, pages 137-146, 1982. 